\title{
Preliminary yield estimation of the 2020 Beirut explosion using video footage from social media
}

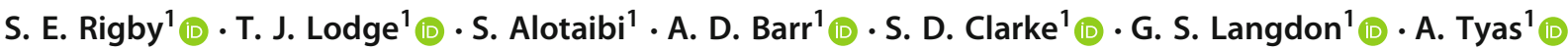

Received: 25 August 2020 / Revised: 1 September 2020 / Accepted: 7 September 2020 / Published online: 22 September 2020

(c) The Author(s) 2020

\begin{abstract}
Rapid, accurate assessment of the yield of a large-scale urban explosion will assist in implementing emergency response plans, will facilitate better estimates of areas at risk of high damage and casualties, and will provide policy makers and the public with more accurate information about the event. On 4 August 2020, an explosion occurred in the Port of Beirut, Lebanon. Shortly afterwards, a number of videos were posted to social media showing the moment of detonation and propagation of the resulting blast wave. In this article, we present a method to rapidly calculate explosive yield based on analysis of 16 videos with a clear line-of-sight to the explosion. The time of arrival of the blast is estimated at 38 distinct positions, and the results are correlated with well-known empirical laws in order to estimate explosive yield. The best estimate and reasonable upper limit of the 2020 Beirut explosion determined from this method are $0.50 \mathrm{kt}$ TNT and $1.12 \mathrm{kt} \mathrm{TNT}$, respectively.
\end{abstract}

Keywords Beirut explosion $\cdot$ Blast $\cdot$ Social media $\cdot$ Time of arrival $\cdot$ Yield estimation

\section{Introduction}

On 4 August 2020, a series of explosions occurred in the Port of Beirut, Lebanon, widely reported to have been caused by detonation of a large quantity of ammonium nitrate (approximately 2750 tonnes) following a fire in the warehouse where it was being stored. The final and largest explosion caused considerable damage to the surrounding area and at the time of writing resulted in at least 181 deaths and over 6000 injuries.

Shortly after the explosion, social media users began sharing videos showing the initial fire, detonation, and propagating blast wave. In many of these videos, the moment of detonation and blast wave time of arrival $\left(t_{\mathrm{a}}\right)$ at the observer's position and/or recognisable landmarks are clearly discernible from the footage and audio. Thus, these videos

Communicated by A. Higgins and E. Timofeev.

Electronic supplementary material The online version of this article (https://doi.org/10.1007/s00193-020-00970-z) contains

supplementary material, which is available to authorized users.

S. E. Rigby

sam.rigby@shef.ac.uk

1 Department of Civil and Structural Engineering, University of Sheffield, Mappin Street, Sheffield S1 3JD, UK make it possible to approximately determine the time of arrival of the shock front at different distances from the source of the explosion.

In this article, we examine 16 videos posted online $[1-16]^{1}$ at various locations across the city of Beirut (Fig. 1). We correlate the calculated distance-time relationship with well-known semi-empirical laws [17] in order to estimate the approximate yield of the 2020 Beirut explosion by minimising the mean absolute error between the data and semi-empirical predictions.

There is a pressing need for rapid, accurate assessment of the size of the explosive yield in such events, both to inform first responders of the likely injuries and structural damage at various distances from the explosion and to provide a factual context for political and media discussion.

\section{Determination of arrival time from video footage}

The videos we analysed in this article were selected having met the following criteria:

\footnotetext{
1 We have attempted to reference a reputable secondary source for those videos where we could not identify the original source.
} 


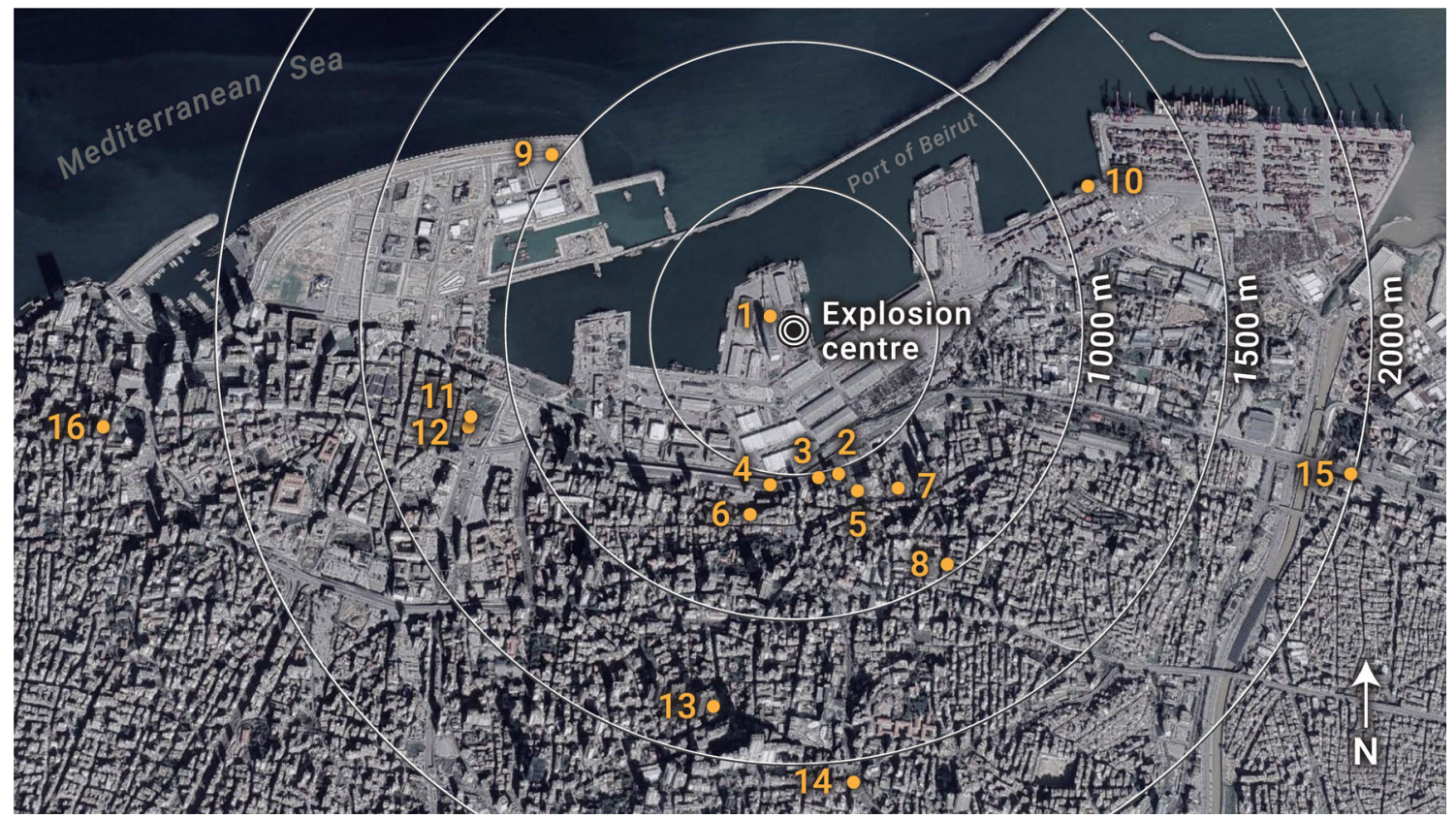

Fig. 1 Filming locations of the 16 videos [1-16] used to estimate the yield of the 2020 Beirut explosion. Satellite imagery from Google Earth (January 2020)

- Direct line-of-sight from the filming location to the source of the explosion in order to determine the moment of detonation ${ }^{2}$, as shown in Fig. 2. This was taken as the start of the frame in which the flash was first visible;

- Identifiable location;

- Synchronised audio and video;

- Filming began before the moment of detonation and continued until after arrival of the blast wave. ${ }^{3}$

The approximate positioning of each video was determined by cross-referencing recognisable buildings and road configurations with Google Street View and satellite images from Google Earth. The distance from the point of detonation (assumed to be the centre of the warehouse) was calculated using the "measure distance" feature in Google Earth. This was taken as the "map distance" rather than "ground distance" (which accounts for changes in elevation) as the height of the filming location was unknown. Owing to uncertainties associated with determining the exact filming location, we present a "best estimate" value as well as a "reasonable upper limit" for each distance (Table 1). Note: videos filmed from a high-rise building have a higher uncertainty in position due

\footnotetext{
$\overline{2}$ In some videos $[11,12,14,16]$, the detonation itself was obscured by neighbouring buildings but could be identified as a clear flash in the images.

3 Video [15] began slightly after detonation, and the time of detonation was estimated by examining the size of the fireball in the first few frames and back extrapolating.
}

to parallax in the satellite images. All distances are measured at street level.

In total, 38 data points were collected from analysis of the social media footage. Three techniques were used for determining time of arrival:

1. Audio In all 16 videos, the arrival of the blast wave could be identified, either as a clear, sharp increase in amplitude of the audio signal (Fig. 2), or by examining the video frame-by-frame for those with higher levels of background noise.

2. Visual In eight videos, the arrival of the blast wave at identifiable intermediate locations was determined by inspecting the video frame-by-frame (15 data points), again see Fig. 2.

3. Fireball In five videos, the size of the detonation product fireball was estimated for a small number of frames after detonation using the nearby grain silo to calibrate the scale of the images. This provided seven additional close-in data points to supplement the distance-time relationship.

Figure 2 shows, as an example, how three arrival times were determined from [5]. The two Visual data points correspond to the time at which the blast was judged to have reached the buildings on the right-hand side and left-hand side of the frame, respectively $(506 \mathrm{~m}$ and $586 \mathrm{~m}$ from the source). The Audio data point was taken as the time to the first local peak in the audio signal. Table 1 provides a summary of the diagnostic techniques used for each video. Again, 


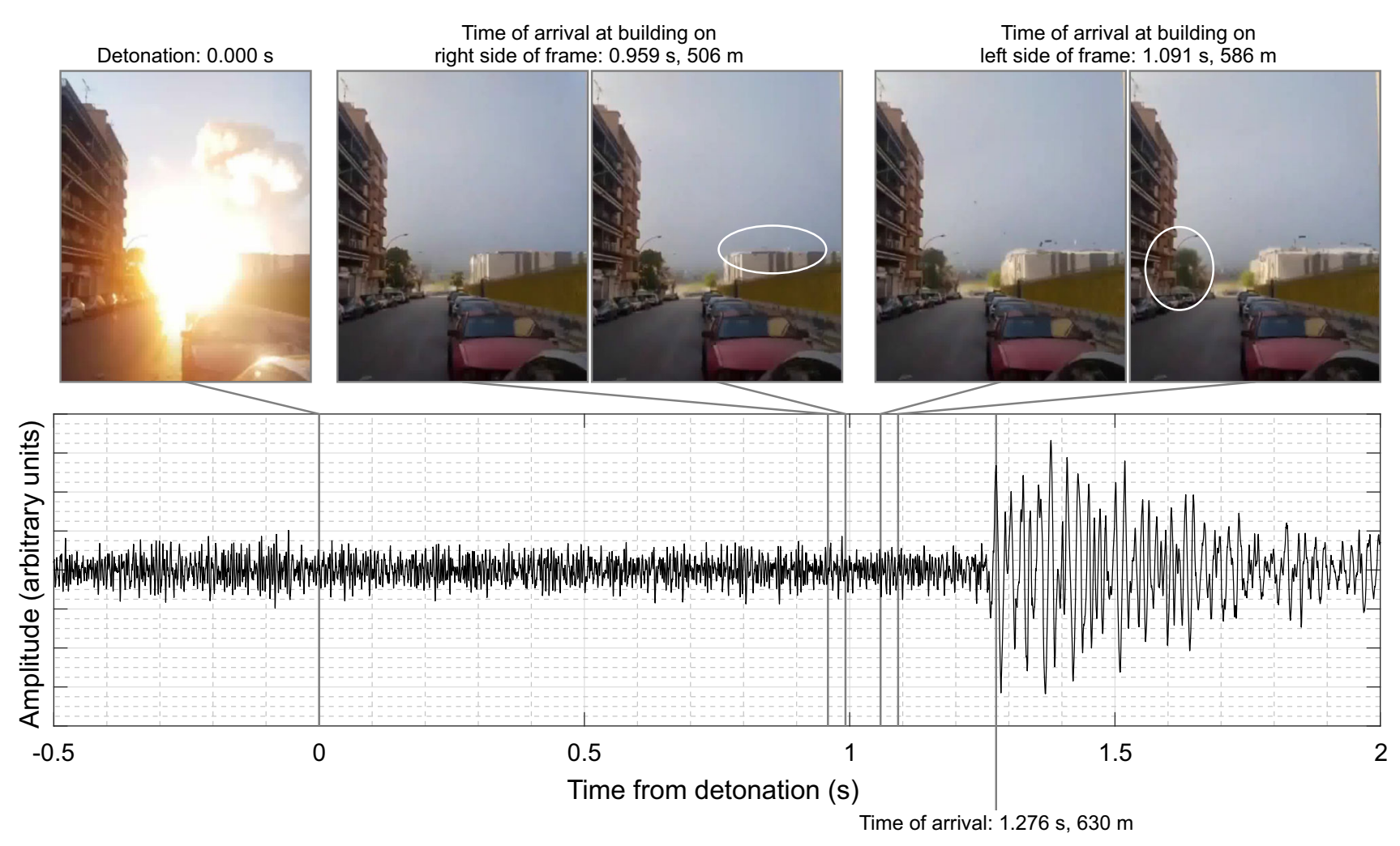

Fig. 2 Example of Visual (top) and Audio (bottom) diagnostics used to determine time of arrival at various locations in video [5]. Note: video stills have been cropped to aid clarity of presentation

we present a best estimate and reasonable upper limit on the arrival times calculated using these methods. The associated timing error was specified as one frame for the Audio method and two frames for Visual and Fireball methods. The frame rate of the videos varied between 24 and $30 \mathrm{fps}$, with resulting minimum and maximum errors of $0.033 \mathrm{~s}(1 / 30 \mathrm{~s})$ and $0.083 \mathrm{~s}(2 / 24 \mathrm{~s})$, respectively.

\section{Estimation of yield from distance-time relationship}

Kingery and Bulmash [17] present semi-empirical relationships between scaled time of arrival, $t_{\mathrm{a}} / W^{1 / 3}$, and scaled distance, $Z=R / W^{1 / 3}$, where $R$ is the distance from the source (in $\mathrm{m}$ ) and $W$ is the TNT-equivalent mass of the explosive (in $\mathrm{kg}$ ). The relationships were derived from the compilation of a large number of experiments which used hemispheres of TNT between 1 and $400,000 \mathrm{~kg}$, detonated on the ground surface and allowed to propagate unobstructed through free air. We present a simplified polylogarithmic function derived by the current authors from the data presented in [17], ${ }^{4}$ with $t_{\mathrm{a}}$ in ms:

\footnotetext{
${ }^{4}$ Coefficient of determination: 0.999 , valid for $0.0674 \leq Z \leq$ $40.0 \mathrm{~m} / \mathrm{kg}^{1 / 3}$.
}

$$
\begin{aligned}
\log \left(t_{\mathrm{a}} / W^{1 / 3}\right)= & 0.0717(\log Z)^{5}-0.0567(\log Z)^{4} \\
& -0.3192(\log Z)^{3}+0.1495(\log Z)^{2} \\
& +1.8165 \log Z-0.3215
\end{aligned}
$$

Equation (1) was solved for $W=0.01-10 \mathrm{kt}$ TNT in increments of $0.01 \mathrm{kt}^{5}$ For each fit, the mean absolute error (MAE) was calculated, with the predicted yield given as the charge mass at the minimum MAE. To allow for direct comparison with the semi-empirical predictions, we have assumed that the explosives were formed into a hemisphere ${ }^{6}$ and that attenuation of the blast wave by the urban environment was negligible. The regression analysis was performed twice: once with the unmodified data (to find the best estimate) and once with the positioning error added to location (including a $10 \mathrm{~m}$ provision for uncertainty in determining the true detonation location) and timing error subtracted from the recorded arrival time, in order to find the reasonable upper limit.

\footnotetext{
5 A constant velocity of $350.7 \mathrm{~m} / \mathrm{s}$ was assumed for $Z \geq 18.0 \mathrm{~m} / \mathrm{kg}^{1 / 3}$ based on reported air temperature and relative humidity of $30^{\circ} \mathrm{C}$ and $66 \%$, respectively, at the time of the explosion. Ambient temperature was assumed to have a negligible effect on arrival time for $Z<18.0 \mathrm{~m} / \mathrm{kg}^{1 / 3}$.

6 This is reasonable, given the approximately hemispherical shape of the fireball shortly after detonation.
} 
Table 1 Summary of videos analysed and diagnostic techniques used for each

\begin{tabular}{|c|c|c|c|c|c|}
\hline \multirow[t]{2}{*}{ References } & \multicolumn{2}{|c|}{ Distance to source $(\mathrm{m})$} & \multicolumn{3}{|c|}{ Diagnostics } \\
\hline & Best estimate & Reasonable upper limit & Audio & Visual & Fireball \\
\hline [1] & 85 & +10 & $\checkmark$ & & \\
\hline [2] & 563 & +10 & $\checkmark$ & $\checkmark$ & \\
\hline [3] & 566 & +30 & $\checkmark$ & & \\
\hline [4] & 572 & +10 & $\checkmark$ & $\checkmark$ & $\checkmark$ \\
\hline [5] & 630 & +30 & $\checkmark$ & $\checkmark$ & \\
\hline [6] & 675 & +30 & $\checkmark$ & $\checkmark$ & \\
\hline [7] & 690 & +10 & $\checkmark$ & $\checkmark$ & \\
\hline [8] & 1000 & +40 & $\checkmark$ & $\checkmark$ & \\
\hline [9] & 1074 & +15 & $\checkmark$ & & \\
\hline [10] & 1120 & +20 & $\checkmark$ & $\checkmark$ & \\
\hline [11] & 1141 & +10 & $\checkmark$ & & $\checkmark$ \\
\hline [12] & 1153 & +10 & $\checkmark$ & $\checkmark$ & $\checkmark$ \\
\hline [13] & 1380 & +20 & $\checkmark$ & & $\checkmark$ \\
\hline [14] & 1605 & +10 & $\checkmark$ & & \\
\hline [15] & 1995 & +40 & $\checkmark$ & & \\
\hline [16] & 2380 & +10 & $\checkmark$ & & $\checkmark$ \\
\hline
\end{tabular}

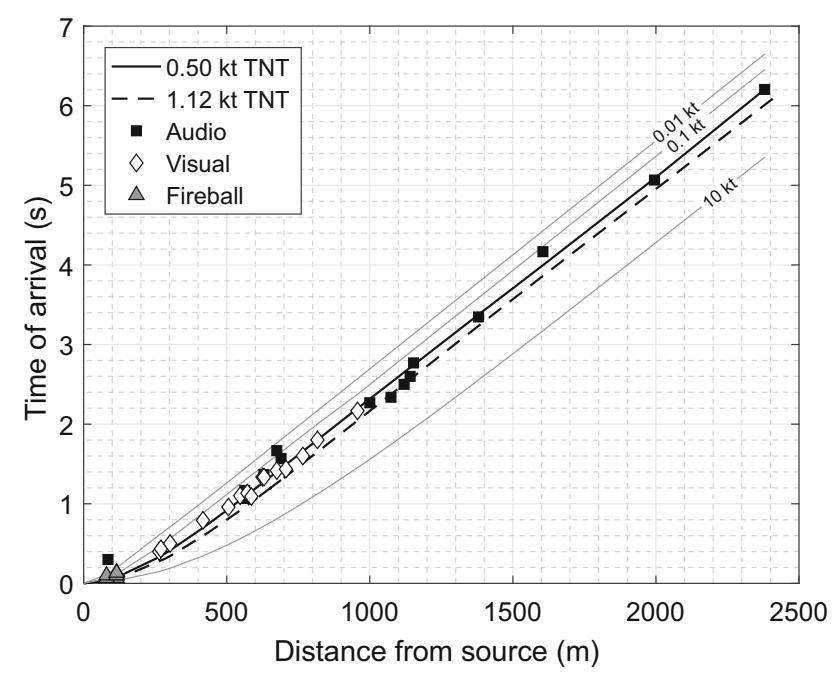

Fig. 3 Collated time of arrival vs distance from analysis of social media video footage, with the best estimate $(0.50 \mathrm{kt} T \mathrm{NT})$ and reasonable upper limit (1.12 kt TNT) curves determined from regression analysis. Also shown are curves for $0.01,0.1$, and $10 \mathrm{kt}$ TNT for reference. Note: error bars are not distinguishable at this scale

Figure 3 shows the compiled data extracted from social media video footage (the radius-time data are also provided as electronic supplementary material) as well as the best estimate $(0.50 \mathrm{kt} \mathrm{TNT})$ and reasonable upper limit $(1.12 \mathrm{kt} \mathrm{TNT})$ curves determined from the regression analysis. These agree remarkably well with the $0.5-1.1 \mathrm{kt}$ range determined from analysis of infrasonic, hydroacoustic, and seismic signals [18]. Also shown are curves for $0.01,0.1$, and $10 \mathrm{kt} \mathrm{TNT}$ to show the effect of order-of-magnitude variations in explo- sive yield on time of arrival. The Audio and Visual data points broadly follow the same trend line, which gives confidence in both approaches.

\section{Closing remarks}

This article presents a method for rapidly determining explosive yield. Here, we extracted time of arrival data from careful examination of video footage uploaded to social media shortly after the 2020 Beirut blast. Despite the videos originating from a range of sources and being located at various points across the city, the resulting distance-time relationship shows a clear trend and is well represented by established semi-empirical predictions for $0.50 \mathrm{kt}$ TNT. In order to account for the uncertainties associated with determining precise locations and timings, the results have also been analysed by taking a reasonable upper limit, which is well represented by semi-empirical predictions for $1.12 \mathrm{kt}$ TNT.

Our initial estimates of explosive yield were $1.0-1.5 \mathrm{kt}$ TNT [19], based on preliminary analysis of the available footage at the time. It was subsequently discovered that some of these videos dropped frames when transferring to or from social media. As more video footage became available, we were able to perform more accurate blast yield predictions with an improved error estimation. This demonstrates the importance of understanding and quantifying sources of error and uncertainty, particularly when considering data with relatively low precision. 
Acknowledgements The authors would like to express their sincere sympathies to all those affected by the events in Beirut on 4 August 2020. The authors would also like to thank those who bravely documented the explosion and uploaded their videos to social media afterwards. Some of the links provided contain distressing content, and viewer discretion is advised.

\section{Compliance with ethical standards}

Conflict of interest The authors declare that they have no conflict of interest. The authors wish to state that referencing a social media profile does not indicate endorsement of the political or other views of that user.

Open Access This article is licensed under a Creative Commons Attribution 4.0 International License, which permits use, sharing, adaptation, distribution and reproduction in any medium or format, as long as you give appropriate credit to the original author(s) and the source, provide a link to the Creative Commons licence, and indicate if changes were made. The images or other third party material in this article are included in the article's Creative Commons licence, unless indicated otherwise in a credit line to the material. If material is not included in the article's Creative Commons licence and your intended use is not permitted by statutory regulation or exceeds the permitted use, you will need to obtain permission directly from the copyright holder. To view a copy of this licence, visit http://creativecomm ons.org/licenses/by/4.0/.

\section{References}

1. https://twitter.com/Darren94775262/status/ 1290720333675352064. Accessed 11 Aug 2020

2. https://twitter.com/Marioam79/status/1291701775339225088. Accessed 11 Aug 2020

3. https://twitter.com/kyf2020/status/1291021170637189126. Accessed 11 Aug 2020

4. https://twitter.com/air_intel/status/1290734832813838337. Accessed 11 Aug 2020
5. https://twitter.com/AllaboutScienc3/status/ 1291412385513652224. Accessed 12 Aug 2020

6. https://twitter.com/Faytuks/status/1291428345993994240. Accessed 11 Aug 2020

7. https://twitter.com/BanTheBBC/status/1290677042426585089. Accessed 11 Aug 2020

8. https://twitter.com/BasedPoland/status/1291574723541962754. Accessed 11 Aug 2020

9. https://streamable.com/zbjj5f. Accessed 12 Aug 2020

10. https://twitter.com/im_Indra07/status/1290730378009796608. Accessed 11 Aug 2020

11. https://twitter.com/ConflictsW/status/1291426642741649410. Accessed 12 Aug 2020

12. https://twitter.com/ConflictsW/status/1290672909875380225. Accessed 11 Aug 2020

13. https://twitter.com/borzou/status/1290675854767513600. Accessed 11 Aug 2020

14. https://twitter.com/Saksham20694519/status/ 1291390141332729862. Accessed 11 Aug 2020

15. https://streamable.com/mcy82f. Accessed 11 Aug 2020

16. https://twitter.com/Mcc_Mark10/status/1290688119675781120. Accessed 11 Aug 2020

17. Kingery, C.N., Bulmash, G.: Airblast parameters from TNT spherical air burst and hemispherical surface burst. Technical Report ARBRL-TR-02555, US Army BRL, Aberdeen Proving Ground, MD, USA (1984)

18. Pilger, C.: Beirut explosion causes strong shock wavesinfrasonic, hydroacoustic and seismic signals registered and investigated by BGR. https://www.seismologie.bgr.de/sdac/erdbeben/ big_quakes/beirut_200804_eng.html. Accessed 24 Aug 2020

19. The terrifying physics behind Beirut's deadly explosion. https://www.wired.co.uk/article/beirut-port-explosion-physics. Accessed 24 Aug 2020

Publisher's Note Springer Nature remains neutral with regard to jurisdictional claims in published maps and institutional affiliations. 